\title{
Nonlinear Target Tracking Algorithm Based on Improved EnPF
}

\author{
Bo Cui
}

\begin{abstract}
An improved ensemble particle filter (EnPF) algorithm combining the advantages of ensemble Kalman filter (EnKF) and particle filter (PF) is proposed for the nonlinear target tracking system. Two independent ensembles are adopted, one ensemble is handled by EnKF first, then the analysis ensemble produced by EnKF and another ensemble is used to generate proposal distribution which approximates the true posterior distribution more accurately, finally $\mathbf{P F}$ is executed to track the target based on this proposal distribution in the nonlinear target tracking system. Simulation results show that the improved EnPF can increase the stability of the tracking system and provide better tracking precision without increasing the computational complexity.
\end{abstract}

Index Terms - Target tracking, ensemble particle filter, proposal distribution, nonlinear

\section{INTRODUCTION}

Many nonlinear tracking algorithms have been advised to the nonlinear target tracking problem before, such as extended Kalman filter(EKF), unscented Kalman filter(UKF), and particle filter(PF), in which PF proposed by Gordon had better tracking performance than others [1]. It was difficult to obtain the true posterior probability density distribution from nonlinear target tracking system, so PF usually uses proposal distribution to simulate the true posterior probability density distribution. If the samples between proposal distribution and the true posterior density distribution exists great bias, the tracking performance of PF will decline quickly. So the key of modifying PF was to find the appropriate proposal distribution function, then many improved PF algorithms based on this thought were proposed in the past ten years. In1998, extended Kalman particle filter(EKPF) proposed by Doucet A [2] used EKF to integrate the latest observation data and approximate the posterior probability density distribution by locally linearized, the tracking performance for nonlinear target tracking system was improved correspondingly. But the large error was introduced when the nonlinear model was linearized by EKF, so the tracking precision was limited in a certain degree. In 2001, unscented particle filter (UPF) [3] proposed by Merwe gained the closer proposal distribution to true posterior probability density distribution, and the tracking performance of UPF was better than EKPF. But with the increase of state dimension, the

Manuscript received July 12, 2016; revised October 13, 2016. This work was supported by national science foundation of P.R.China under Grant No. 61271341 and technology innovation project of Southwest Jiaotong University under Grant No. 2682015CX074.

The author is with School of Information Science and Technology, Southwest Jiaotong University, Chengdu 611756, China (e-mail: bcui@swjtu.edu.cn). calculation of UPF rose rapidly, and the performance of UPF was limited by the assumption of Gaussian noise distribution in addition [4]. In 1994, Geir Evensen proposed the ensemble Kalman filter (EnKF) [5], which was a particle filter algorithm introducing ensemble forecast thought and integrated the advantages of ensemble forecast theory and Kalman filter algorithm. EnKF was the general form of UKF, which used ensemble forecasted thought to replace deterministic sampling of UKF and formed the observation ensemble. So the EnKF can be used more widely than UKF and become the better choice to propagate the proposal distribution, but EnKF was also limited by the assumption of Gaussian noise distribution in a certain degree. In 2006, Werrts and El Serafy compared the EnKF and PF in detail [6]. Paper [7] proposed ensemble particle filter (EnPF) and presented several combinations of EnKF and PF, and then the parallel mode of EnKF and PF was applied to rainfall-runoff model. In 2009, Mandel [8] used an EnKF as predicator at first, and then used PF as corrector to reduce the affection of Gaussian distribution assumption of EnKF. In 2011, DU Hangyuan used EnKF to propagate the proposal distribution of $\mathrm{PF}$, and the proposal distribution function integrated the latest observation into state transition density of the system, so the proposal distribution can approximate the true posterior distribution more accurately [9]. The improved EnPF for nonlinear target tracking system is proposed in this paper. Two independent ensembles are adopted, one ensemble is handled by EnKF first, then the analysis ensemble produced by EnKF and another ensemble is used to generate proposal distribution of $\mathrm{PF}$, finally $\mathrm{PF}$ is executed based on this proposal distribution [10], [11]. The improved EnPF not only combines the advantages of EnKF and PF, but also solves the deficiency of Gaussian noise distribution of EnKF and provides better tracking performance under the nonlinear condition.

\section{THE STRUCTURE OF IMPROVED ENPF}

EnPF combines the advantages of EnKF and PF. PF uses a set of weighted particles to describe any posterior probability distribution function, but there is no updating in nature because of only changing the weights of particles. There are several combinations of EnKF and PF as follows:

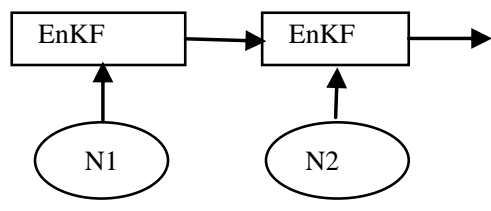

Fig. 1. EnKF pre-filter. 
It is pre-filter structure in Fig. 1, two independent ensembles are included. The number of members in the first ensemble is $\mathrm{N} 1$, and the number of members in the second ensemble is N2. The first ensemble with N1 members uses EnKF to pre-filter, and then the state estimation after pre-filter is as input for the second EnKF filter using the ensemble with $\mathrm{N} 2$ members.

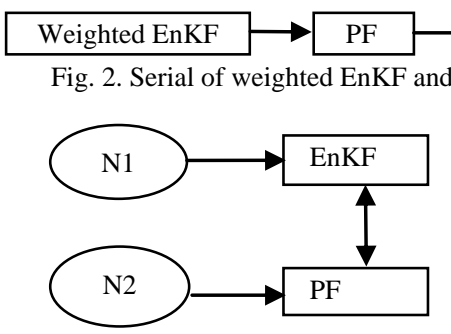

Fig. 3. Parallel of EnKF and PF.

Fig. 2 uses weighted EnKF filter to estimate and output the weighted average, then the PF is executed to track the target, in which EnKF computes corresponding weight using the similar method to PF for each ensemble member.

Fig. 3 describes the parallel structure of EnKF and PF. The ensemble with N1 members is executed by EnKF, and then the ensemble with $\mathrm{N} 2$ members is handled by PF. Whether EnKF and PF interacting each other depends on the specific system.

The method in this article is based on the first and second structure. Two independent ensembles are adopted, the first ensemble with $\mathrm{N} 1$ members is handled by EnKF at first, then the analysis ensemble of EnKF and second set with N2 particles constitute the proposal distribution of $\mathrm{PF}$, following random sampling is done from this proposal distribution, finally $\mathrm{PF}$ is executed to track the target. The structure of improved EnPF is described in Fig. 4.

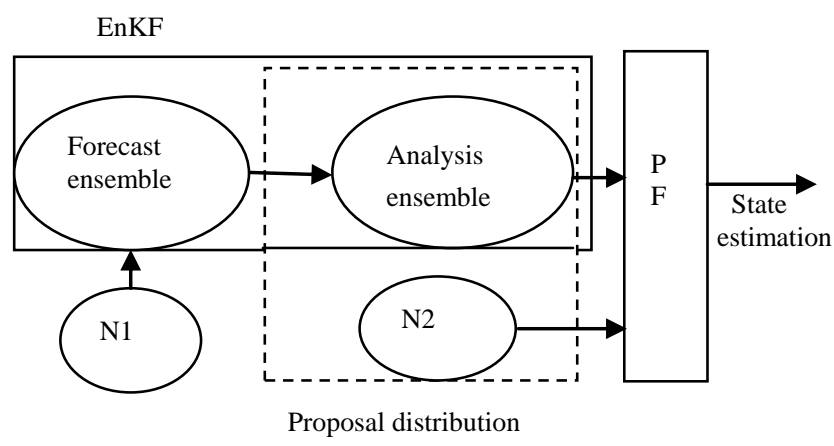

Fig. 4. The structure of improved EnPF.

\section{NONLINEAR TARGET TRACKING ALGORITHM BASED ON IMPROVED ENPF}

Consider a discrete-time nonlinear system with dynamics

$$
X_{i}=f\left(X_{i-1}\right)+W_{i}
$$

and measurements

$$
Z_{i}=H\left(X_{i}\right)+N_{i}
$$

where $W_{i}$ is the process noise and model error, and $N_{i}$ is the measurement noise, $Z_{i}$ is the measurement vector in the target tracking system.

1) Initialing: The initial ensemble is produced by prior knowledge. At time k, the ensemble can be described as:

$$
X_{i}^{R}(k)=\left|\begin{array}{ccccc}
x_{1}(k) & x_{2}(k) & x_{i}(k) & \ldots & x_{N 1}(k) \\
y_{1}(k) & y_{2}(k) & y_{i}(k) & \ldots & y_{N 1}(k) \\
z_{1}(k) & z_{2}(k) & z_{i}(k) & \ldots & z_{N 1}(k) \\
\dot{x}_{1}(k) & \dot{x}_{2}(k) & \dot{x}_{i}(k) & \ldots & \dot{x}_{N 1}(k) \\
\dot{y}_{1}(k) & \dot{y}_{2}(k) & \dot{y}_{i}(k) & \ldots & \dot{y}_{N 1}(k) \\
\dot{z}_{1}(k) & \dot{z}_{2}(k) & \dot{z}_{i}(k) & \ldots & \dot{z}_{N 1}(k)
\end{array}\right|
$$

where $\mathrm{N} 1$ is the number of ensemble members $X_{i}^{R}(k)=\left[x_{i}(k), y_{i}(k), z_{i}(k), \dot{x}_{i}(k), \dot{y}_{i}(k), \dot{z}_{i}(k)\right]^{T}$ is state of the $\mathrm{i}$-th member at time $\mathrm{k}, \mathrm{R}=\mathrm{F}$ is forecasted state, $\mathrm{R}=\mathrm{A}$ is analysis state.

2) EnKF sampling

- Forecasted step: forecasted state of each ensemble member is computed at time $\mathrm{k}+1$ :

$$
X_{i}^{F}(k+1)=f\left(X_{i}^{A}(k)\right)+W_{i}(k)
$$

and the forecasted state can be described as:

$$
\overline{X^{F}(k+1)}=\sum_{i=1}^{N} w_{i}(k) X_{i}^{F}(k+1)
$$

where $\sum_{i=1}^{N} w_{i}(k)=1$.

- Kalman gain matrix $K(k+1)$ is computed at time $\mathrm{k}+1$ :

$$
K(k+1)=P^{F}(k+1) H^{T}\left(H P^{F}(k+1) H^{T}+R_{i}\right)^{-1}
$$

- Analysis step: Observed data are used at the analysis step, and state variable and error covariance matrix are computed at time $\mathrm{k}+1$. For $\mathrm{i}=1 \ldots \mathrm{N}$,

$$
\begin{aligned}
& X_{i}^{A}(k+1)=X_{i}^{F}(k+1)+K(k+1)\left\lfloor Z_{i}(k+1)-H\left(X_{i}^{F}(k+1)\right)\right\rfloor \\
& \overline{X^{A}(k+1)}=\sum_{i=1}^{N} w_{i}(k) X_{i}^{A}(k+1) \\
& P^{A}(k+1)=\sum_{i=1}^{N} w_{i}(k)\left(X_{i}^{A}(k+1)-\overline{X^{A}(k+1)}\right)\left(X_{i}^{A}(k+1)-\overline{X^{A}(k+1)}\right)^{T}
\end{aligned}
$$

3) The proposal distribution is generated from the ensemble with N2 particles and the analysis ensemble of EnKF.

$$
q=\left\{N\left(X_{i}^{A}(k+1), P_{i}^{A}(k+1)\right), N 2\right\}
$$

4) Random sampling is done from proposal distribution function $\mathrm{q}$, and the new samplings are obtained at time $\mathrm{k}+1$

$$
X_{i}(k+1) \sim q\left(X_{i}(k+1) \mid X_{i}(k), Z(k+1)\right)
$$

5) Computing the weight of each particle

$$
w_{i}(k+1)=w_{i}(k) \frac{p\left(Z_{i}(k+1) \mid X_{i}(k+1)\right) p\left(X_{i}(k+1) \mid X_{i}(k)\right)}{q\left(X_{i}(k+1) \mid X_{i}(k), Z(k+1)\right)}
$$


6) Weight normalizing:

$$
w_{i}(k+1)=\frac{w_{i}(k+1)}{\sum_{j=1}^{N} w_{j}(k+1)}
$$

7) Resampling:

- Use $\hat{N}_{\text {eff }}=\left(\sum_{i=1}^{N_{s}} w_{i}(k+1)\right)^{-1}$ to compute the number of effective particles of PF;

- If the number of the effective particles is less than the threshold, then resample $\mathrm{N}$ random particles

8) Fusion results:

$$
\begin{aligned}
& \hat{x}(k+1)=\sum_{i=1}^{N} w_{i+1}(k+1) X_{i}(k+1) \\
& P(k+1)=\sum_{i=1}^{N} w_{i+1}(k+1)\left(X_{i}(k+1)-\right. \\
& \left.\hat{X}_{i}(k+1)\right)\left(X_{i}(k+1)-\hat{X}_{i}(k+1)\right)^{T}
\end{aligned}
$$

9) Go to step 2).

\section{EXPERIMENTS AND ANALYSIS}

\section{A. Experiments}

Fig. 5 shows the comparison of different nonlinear target tracking algorithms for one random nonlinear motion. Fig. 6 shows the comparison of different nonlinear target tracking algorithms for one random nonlinear motion. Fig. 6 shows the comparison of different nonlinear target tracking algorithms for another random nonlinear motion. The feasibility of improved EnPF algorithm can be observed in the nonlinear target tracking system when the target performs the motion.

From the figures we can see that UKF, EnKF, PF, EnPF can be applied to nonlinear target tracking system. But UKF has the lowest performance due to the limitation of non-Gaussian noise distribution condition. The performance of EnKF is a little better than the UKF because EnKF is the extension of UKF, but still affected in a certain degree by the assumption of non-Gaussian noise. PF can be applied to any nonlinear non-Gaussian target tracking system, but when the selection of proposal distribution is unreasonable, PF still exist certain tracking error.

It can be observed from figures that improved EnPF have the better tracking performance than UKF, PF, and EnKF. EnPF is the improvement of PF and utilizes the EnKF to produce the proposal distribution function of PF. From the theoretical analysis and experiments, the proposal distribution function of improved EnPF is generated based on the analysis ensemble and another set with N2 particles, which solves the limitation of Gaussian system for EnKF. So the tracking performance of EnPF based on EnKF is better than the performance of UPF based on UKF.
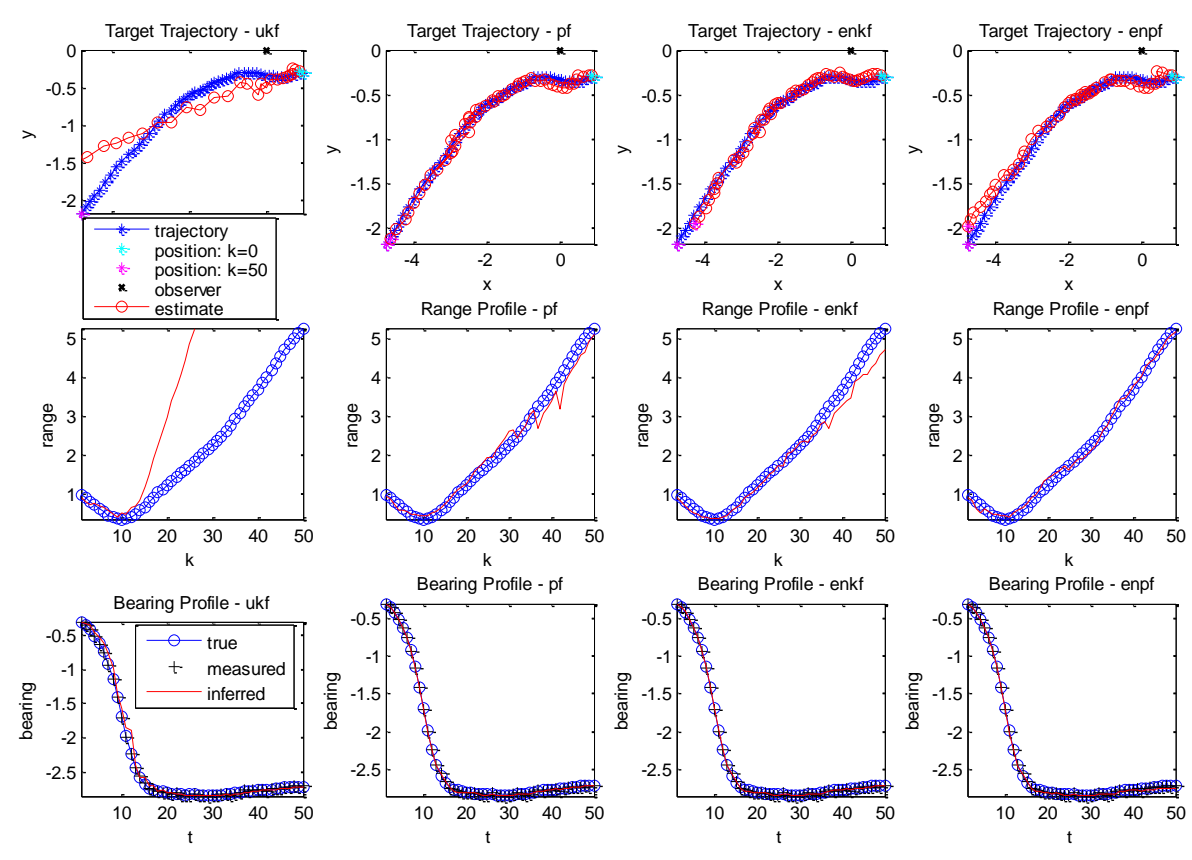

Fig. 5. Comparison of different nonlinear target tracking algorithms.

\section{B. Characteristics Analysis}

1) The improved EnPF adopts two independent ensembles, where the ensemble with $\mathrm{N} 1$ members is handled by EnKF, and the ensemble with $\mathrm{N} 2$ members is filtered by PF. The algorithm can increase the stability of the tracking system. Once PF does not work normally, EnKF can filter independently using the ensemble with
N1 members. If EnKF disables, PF tracks the object independently. Both EnKF and PF work normally, the tracking performance is better than EnKF or PF alone, so the improved EnPF can increase the stability.

2) The nonlinear target tracking algorithm of improved EnPF does not use directly the state estimation of EnKF. It generates the proposal distribution from the analysis 
ensemble of EnKF and another set with N2 particles, and random sampling is done from this proposal distribution. So the proposal distribution is closer to true distribution and don't limited by the condition of Gaussian noise distribution. The improved EnPF can apply to non-Gaussian system which solving the problem of
EnKF limited by the Gaussian noise assumption.

3) The improved EnPF fuses the latest measurements at analysis step, which remedies the defection of only changing the weights of particles in $\mathrm{PF}$ in which no really updating is executed.
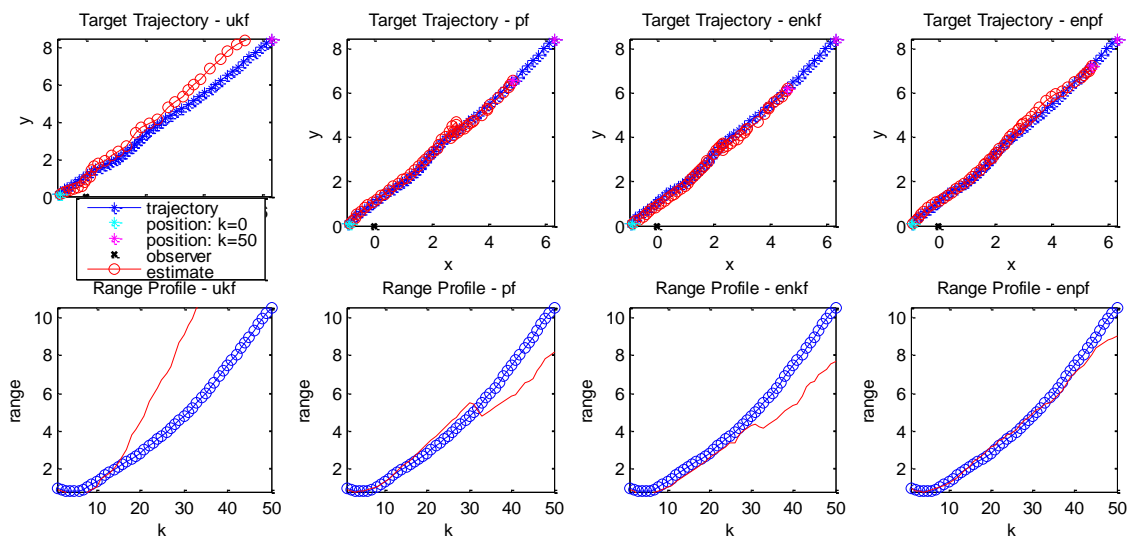

Range Profile - $p$
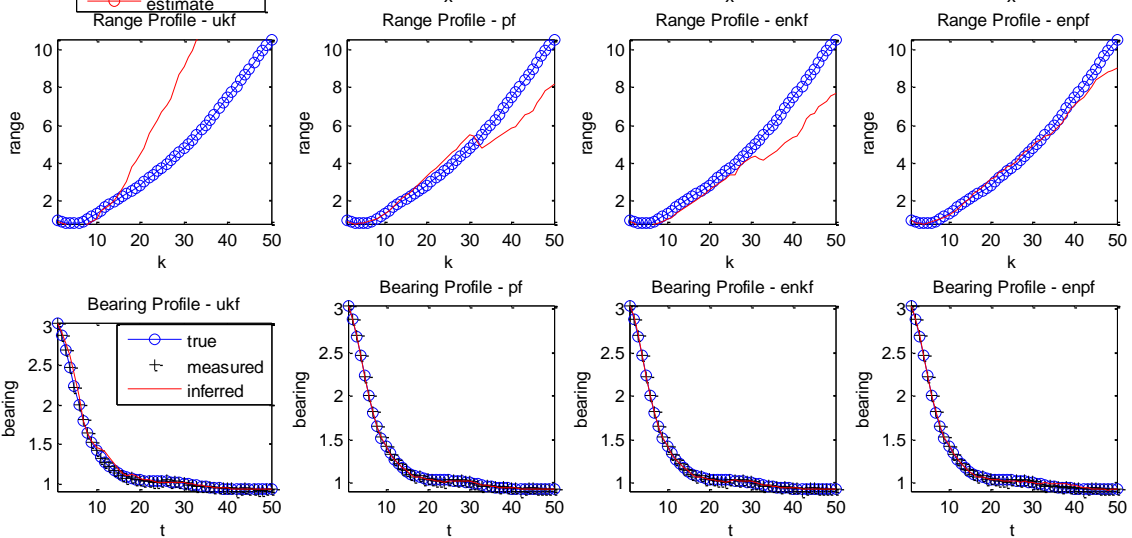

Fig. 6. Comparison for the another random nonlinear motion.

\section{Comparison of Computation Complexity}

Supposing a multiplication is executed in one forecasted step of EKF, and B multiplication is executed in one analysis step of EKF. There are $2 n+1$ sample in UKF, $N$ particles in $\mathrm{PF}, \mathrm{N} 1$ members in the ensemble of EnKF, and $\mathrm{k}$ Monte Carlo for generating observed ensemble. And EkPF is the combination of EnKF and PF, UPF is integration of UKF and $\mathrm{PF}$, EnPF is the combination of EnKF and PF. Then comparison of different nonlinear filter algorithms is described in Table I. From table we can see that EnPF has the same computation complexity as EkPF and UPF but has higher tacking precision.

TABLE I: COMPARISON OF NONLINEAR FILTER ALGORITHMS

\begin{tabular}{ll}
\hline \hline Algorithm & \multicolumn{1}{c}{ Computation complexity } \\
\hline UKF & $4 n^{3}+(4+5 m) n^{2}+(6 n+2) m^{2}+5 m n+(2 n+1) *(A+B)$ \\
PF & $N^{*}(A+B)$ \\
EnKF & $4 n^{3}+(4+5 m) n^{2}+(6 n+2) m^{2}+5 m n+k N_{1}+N_{1}(A+B)$ \\
EkPF & $N^{*}\left(3 n^{3}+3 m n^{2}+2 m^{2} n+m n+A+B\right)$ \\
$\mathrm{UPF}$ & $N^{*}\left(4 n^{3}+(4+5 m) n^{2}+(6 n+2) m^{2}+5 m n+(2 n+1) *(A+B)+B\right)$ \\
EnPF & $4 n^{3}+(4+5 m) n^{2}+(6 n+2) m^{2}+5 m n+k N_{1}+N_{1}(A+B)+N A+N B$ \\
\hline \hline
\end{tabular}

\section{CONCLUSION}

An improved EnPF combining the advantages of EnKF and $\mathrm{PF}$ has been presented in the nonlinear target tracking system. The algorithm can increase the stability of the tracking system, and generates the proposal distribution closer to the true probability density distribution. Furthermore, the improved EnPF has the better tracking performance without increasing the computation complexity.

\section{ACKNOWLEDGMENT}

This work is supported by national science foundation of P.R.China under Grant No. 61271341 and technology innovation project of Southwest Jiaotong University under Grant No. 2682015CX074.

\section{REFERENCES}

[1] N. Gordon and D. Salmond, "Novel approach to non-linear and non-Gaussian bayesian state estimation," Radar and Signal Processing, Proc of Institute Electirc Engineering, vol. 140, pp. 107-113, May 1993.

[2] A. Doucet, S. Godsill, and C. Andrieu, "On sequential monte carlo methods for bayesian filtering," Statistics and Computing, vol. 10, pp. 197-208, July 2000.

[3] R. V. D. Merwe, A. Doucet, N. D. Freitas, and E. Wan, "The unscented particle filter," Advances in Neural Information Processing Systems, vol. 13, pp. 584-590, Jan. 2001.

[4] X. Luo and I. M. Moroz, "Ensemble Kalman filter with the unscented transform," Physical D: Nonlinear Phenomena, vol. 238, pp. 549-562, March 2009.

[5] G. Evensen, "Sequential data assimilation with a nonlinear quasigeostrophic model using monte carlo methods to forecast error statistics," Journal of Geophysical Research, vol. 99, pp. 10143-10162, May 1994.

[6] A. H. Weerts, "Particle filtering and ensemble Kalman filtering for state updating with hydrological conceptual rainfall-runoff models," Water Resources Research, vol. 42, pp. 123-154, Sep. 2006.

[7] G. V. Delft, G. Y. Serafy, and A. W. Heemink, "The ensemble particle filter (EnPF) in rainfall-runoff models," Stochastic Environmental Research and Risk Assessment, vol. 23, pp. 1203-1211, Jan. 2009.

[8] J. andel and J. D. Beezley. An Ensemble Kalman-Particle Predictor-Corrector Filter for Non-gaussian Data Assimilation, Springer Berlin Heidelberg, vol. 55, pp. 470-478, 2009.

[9] H. Y. Du, Y. L. Hao, and Y. X. Zhao, "Improved particle filter based on ensemble Kalman," Systems Engineering and Electronics, vol. 33, pp. 1653-1657, 2011. 
[10] Z. T. Hu, J. Zhang, and G. Zhen, "Multi-sensor ensemble Kalman filtering algorithm based on observation fuzzy support degree fusion," International Journal for Light and Electron, vol. 127, pp. 8520-8529, Oct. 2016.

[11] N. E. Apriliani and H, Nurhadi, "Ensemble and fuzzy Kalman filter for position estimation of an autonomous underwater vehicle based on dynamical system of AUV motion," Expert Systems with Applications, vol. 68, pp. 29-35, Feb. 2017.

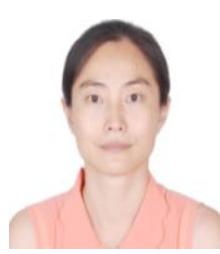

Bo Cui was born in Heilongjiang province, China, in Aug, 1979. She received the B.S. degree in 2001 and the M.S. degree in 2004, both in computer software from Information Science and Technology School, Jilin University, Changchun, and received her Ph.D. degree in signal and information from the Information

University in 2012.
She has participated in 2 NSFC projects, and presided over a number of vertical and horizontal scientific research projects. She has published more than 10 research papers on high-quality journals and conferences. Such as:

Bo Cui. A method of sensor assignment for tracking multi-target, Journal of Jilin university (engineering and technology Edition), 2009, 39(4): 1115-1118. Track Fusion Algorithm Based on Ensemble Kalman Filter. International Journal of Advancements in Computing Technology, 175-184 Her research interests include nonlinear signal processing, target tracing and information fusion.

Dr. Cui is the member of information security expert group of Sichuan Province. 